\title{
Indirect Performance Sensing for On-Chip Analog Self-Healing via Bayesian Model Fusion
}

\author{
S. Sun ${ }^{1}$, F. Wang ${ }^{1}$, S. Yaldiz ${ }^{1}$, X. Li ${ }^{1}$, L. Pileggi ${ }^{1}$, A. Natarajan ${ }^{2,3}$, M. Ferriss ${ }^{2}$, J. Plouchart ${ }^{2}$, B. Sadhu ${ }^{2}$, B. Parker ${ }^{2}$, A. \\ Valdes-Garcia $^{2}$, M. Sanduleanu ${ }^{2}$, J. Tierno ${ }^{2}$, D. Friedman ${ }^{2}$ \\ ${ }^{1}$ Electrical \& Computer Engineering Department, Carnegie Mellon University, Pittsburgh, PA, USA, 15213 \\ ${ }^{2}$ IBM T. J. Watson Research Center, Yorktown Heights, NY, USA, 10598 \\ ${ }^{3}$ Electrical \& Computer Engineering Department, Oregon State University, Corvallis, OR, USA, 97331
}

\begin{abstract}
On-chip analog self-healing requires low-cost sensors to accurately measure various performance metrics. In this paper, we propose a novel approach of indirect performance sensing based upon Bayesian model fusion (BMF) to facilitate inexpensive-yet-accurate on-chip performance measurement. A 25GHz differential Colpitts voltage-controlled oscillator (VCO) designed in a $32 \mathrm{~nm}$ CMOS SOI process is used to validate the proposed indirect performance sensing and self-healing methodology. Our silicon measurement results demonstrate that the parametric yield of the VCO is improved from $0 \%$ to $69.17 \%$ for a wafer after the proposed self-healing is applied.
\end{abstract}

\section{INTRODUCTION}

With the aggressive scaling of nanoscale integrated circuit (IC) technology, large-scale process variation is a critical issue for today's analog ICs [1]-[3]. As the traditional over-design technique becomes impractical, on-chip self-healing has emerged as a promising methodology to address the variability issue [4]-[5]. The key idea of self-healing is to actively monitor the post-manufacturing circuit performance metrics and then adaptively adjust a number of tuning knobs (e.g., bias current) in order to meet the given performance specifications.

To practically implement on-chip self-healing, a large number of analog performance metrics must be measured accurately and inexpensively by on-chip sensors. Such a measurement task, however, is not trivial, because many analog performance metrics (e.g., phase noise) cannot be easily measured by on-chip sensors. For this reason, the idea of indirect performance sensing has been recently proposed [6]-[7] where the performance of interest $(\mathrm{PoI})$ is not directly measured by an on-chip sensor. Instead, it is accurately predicted from a set of other performance metrics, referred to as the performances of measurement (PoM), that are highly correlated with PoI and are easy to measure.

Towards this goal, indirect sensor modeling is a critical task where the objective is to build a mathematical model to capture the correlation between PoI and PoM so that PoI can be predicted from PoM. These models must be repeatedly recalibrated to accommodate the process shift associated with manufacturing lines. While efficient modeling methods have been explored in the literature [6], the aforementioned model re-calibration issue has not been extensively studied yet. Hence, there is a need to develop a new methodology to facilitate efficient model re-calibration with low cost (i.e., requiring few additional measurement data). As such, the overhead of indirect performance sensing and, eventually, the overhead of analog self-healing can be minimized.
In this paper, a novel Bayesian model fusion (BMF) technique is proposed to address the model re-calibration problem and, hence, make analog self-healing of practical utility. The key idea of BMF is to combine the old (i.e., before process shift) indirect sensor model with very few new (i.e., after process shift) measurement data to generate a new model that is aligned with the new process conditions. Mathematically, the old model is encoded as prior knowledge, and a Bayesian inference is derived to optimally fit the new model by maximum-a-posteriori (MAP) estimation.

Furthermore, an analog self-healing flow is developed where the indirect sensor models are extracted by the proposed BMF technique. The proposed self-healing flow is validated for a $25 \mathrm{GHz}$ differential Colpitts VCO designed in a $32 \mathrm{~nm}$ CMOS SOI process. Our silicon measurement results demonstrate that the parametric yield of the VCO is improved from $0 \%$ to $69.17 \%$ for a wafer after self-healing is applied.

The remainder of this paper is organized as follows. In Section II, we review indirect performance sensing and then derive the BMF method in Section III. The proposed BMFbased self-healing flow is described and validated for a VCO example in Section IV. Finally, we conclude in Section V.

\section{INDIRECT PERFORMANCE SENSING}

We denote a PoI as $f$ and the PoM as:

$$
\mathbf{x}=\left[\begin{array}{llll}
x_{1} & x_{2} & \cdots & x_{M}
\end{array}\right]^{T},
$$

where $M$ stands for the number of performance metrics belonging to PoM. The objective of indirect performance sensing is to accurately predict the PoI $f$ from the PoM $\mathbf{x}$ that is highly correlated with $f$ and can be easily measured by onchip sensors. To this end, we need to approximate $f$ as an analytical function of $\mathbf{x}$ :

$$
f(\mathbf{x})=\sum_{k=1}^{K} \alpha_{k} \cdot b_{k}(\mathbf{x}),
$$

where $\left\{b_{k}(\mathbf{x}) ; k=1,2, \ldots, K\right\}$ contains the basis functions (e.g., linear and quadratic polynomials), $\left\{\alpha_{k} ; k=1,2, \ldots, K\right\}$ contains the model coefficients, and $K$ is the total number of basis functions. Once the model $f(\mathbf{x})$ in (2) is determined, it can be used to predict the PoI $f$ from the on-chip measurements of the PoM $\mathbf{x}$.

In general, a number of silicon chips are required to fit the model $f(\mathbf{x})$ in (2). The PoM (i.e., $\mathbf{x})$ values of these chips are measured by on-chip sensors and the corresponding PoI (i.e., f) values are measured by an off-chip tester. These measurement data are then used to determine the model 
coefficients $\left\{\alpha_{k} ; k=1,2, \ldots, K\right\}$ in (2) by ordinary leastsquares (OLS) fitting [8]. More details about the traditional OLS method can be found in [8].

\section{BAYESIAN MODEL FUSION}

Our proposed BMF method aims to efficiently re-calibrate the indirect sensor model $f(\mathbf{x})$ in (2) with consideration of process shift. Towards this goal, we consider two different models: the old model $f_{O L D}(\mathbf{x})$ and the new model $f_{N E W}(\mathbf{x})$ :

$$
\begin{gathered}
f_{O L D}(\mathbf{x})=\sum_{k=1}^{K} \alpha_{O L D, k} \cdot b_{k}(\mathbf{x})+\varepsilon_{O L D} \\
f_{N E W}(\mathbf{x})=\sum_{k=1}^{K} \alpha_{N E W, k} \cdot b_{k}(\mathbf{x})+\varepsilon_{N E W},
\end{gathered}
$$

where $\left\{\alpha_{O L D, k} ; k=1,2, \ldots, K\right\}$ and $\left\{\alpha_{N E W, k} ; k=1,2, \ldots, K\right\}$ contain the old and new model coefficients respectively, and $\varepsilon_{O L D}$ and $\varepsilon_{N E W}$ denote the modeling error associated with the old and new models respectively.

The old model $f_{O L D}(\mathbf{x})$ in (3) is fitted before process shift occurs. Hence, we assume that the old model coefficients $\left\{\alpha_{O L D, k} ; k=1,2, \ldots, K\right\}$ are already known, before fitting the new model $f_{N E W}(\mathbf{x})$ in (4). The objective of BMF is to accurately determine the new model coefficients $\left\{\alpha_{N E W, k} ; k=\right.$ $1,2, \ldots, K\}$ by combining the old model coefficients $\left\{\alpha_{O L D, k} ; k\right.$ $=1,2, \ldots, K\}$ with very few new measurement data.

Our proposed BMF method consists of two major steps: (i) statistically extracting the prior knowledge from the old model coefficients $\left\{\alpha_{O L D, k} ; k=1,2, \ldots, K\right\}$ and encoding it as a prior distribution, and (ii) optimally determining the new model coefficients $\left\{\alpha_{N E W, k} ; k=1,2, \ldots, K\right\}$ by MAP estimation. In what follows, we will describe these two steps in detail.

\section{A. Prior Knowledge Definition}

Since the two models $f_{O L D}(\mathbf{x})$ and $f_{N E W}(\mathbf{x})$ in (3)-(4) both approximate the mathematical mapping from PoM to PoI, we expect that the model coefficients $\left\{\alpha_{O L D, k} ; k=1,2, \ldots, K\right\}$ and $\left\{\alpha_{N E W, k} ; k=1,2, \ldots, K\right\}$ are similar. On the other hand, $f_{O L D}(\mathbf{x})$ and $f_{N E W}(\mathbf{x})$ cannot be exactly identical due to process shift. To statistically encode the "common" information between $f_{O L D}(\mathbf{x})$ and $f_{N E W}(\mathbf{x})$, we define a Gaussian distribution as our prior distribution for each new model coefficient $\alpha_{N E W, k}$ :

$$
p d f\left(\alpha_{N E W, k}\right) \sim \operatorname{Gauss}\left(\alpha_{O L D, k}, \lambda^{2} \cdot \alpha_{O L D, k}^{2}\right) \quad(k=1,2, \cdots, K),
$$

where $\alpha_{O L D, k}$ and $\lambda^{2} \cdot \alpha_{O L D, k}{ }^{2}$ are the mean and variance of the Gaussian distribution respectively, and $\lambda$ is a parameter that can be determined by cross-validation [8]-[9].

The prior distribution in (5) has a two-fold meaning. First, the Gaussian distribution $p d f\left(\alpha_{N E W, k}\right)$ is peaked at its mean value $\alpha_{N E W, k}=\alpha_{O L D, k}$, implying that the old coefficient $\alpha_{O L D, k}$ and the new coefficient $\alpha_{N E W, k}$ are likely to be similar. In other words, since the Gaussian distribution $p d f\left(\alpha_{N E W, k}\right)$ exponentially decays with $\left(\alpha_{N E W, k}-\alpha_{O L D, k}\right)^{2}$, it is unlikely to observe a new coefficient $\alpha_{N E W, k}$ that is extremely different from the old coefficient $\alpha_{O L D, k}$. Second, the standard deviation of the prior distribution $p d f\left(\alpha_{N E W, k}\right)$ is proportional to $\left|\alpha_{O L D, k}\right|$. It means that the absolute difference between the new coefficient $\alpha_{N E W, k}$ and the old coefficient $\alpha_{O L D, k}$ can be large (or small), if the magnitude of the old coefficient $\left|\alpha_{O L D, k}\right|$ is large (or small). Restating in words, each new coefficient $\alpha_{N E W, k}$ has been provided with a relatively equal opportunity to deviate from the corresponding old coefficient $\alpha_{O L D, k}$.

To complete the definition of the prior distribution for all new model coefficients $\left\{\alpha_{N E W, k} ; k=1,2, \ldots, K\right\}$, we further assume that these coefficients are statistically independent and their joint distribution is represented as:

$$
p d f\left(\boldsymbol{\alpha}_{N E W}\right)=\prod_{k=1}^{K} p d f\left(\alpha_{N E W, k}\right),
$$

where $\boldsymbol{\alpha}_{N E W} \in \mathfrak{R}^{K}$ is a vector containing all new coefficients $\left\{\alpha_{N E W, k} ; k=1,2, \ldots, K\right\}$. The independence assumption in (6) simply implies that we do not know the correlation information among these coefficients as our prior knowledge. The correlation information will be learned from the new measurement data, when the posterior distribution is calculated by MAP estimation in the next sub-section.

\section{B. Maximum-A-Posteriori Estimation}

Once the prior distribution is defined, we collect a few new measurement data $\left\{\left(\mathbf{x}^{(n)}, f_{N E W}^{(n)}\right) ; n=1,2, \ldots, N\right\}$, where $\mathbf{x}^{(n)}$ and $f_{N E W}{ }^{(n)}$ are the values of $\mathbf{x}$ and $f_{N E W}(\mathbf{x})$ at the $n$-th data point respectively after process shift occurs. These new measurement data can tell us additional information about the process shift and, hence, help us to determine the new coefficients $\left\{\alpha_{N E W, k} ; k=1,2, \ldots, K\right\}$.

Based on Bayes' theorem [8]-[9], the uncertainties of the new coefficients $\left\{\alpha_{N E W, k} ; k=1,2, \ldots, K\right\}$ after knowing the data $\left\{\left(\mathbf{x}^{(n)}, f_{N E W}^{(n)}\right) ; n=1,2, \ldots, N\right\}$ can be mathematically described by the following posterior distribution:

$$
p d f\left(\boldsymbol{\alpha}_{N E W} \mid \mathbf{f}_{N E W}\right) \propto p d f\left(\boldsymbol{\alpha}_{N E W}\right) \cdot p d f\left(\mathbf{f}_{N E W} \mid \boldsymbol{\alpha}_{N E W}\right),
$$

where $\mathbf{f}_{N E W} \in \mathfrak{R}^{N}$ is a vector with the new data $\left\{f_{N E W}{ }^{(n)} ; n=1\right.$, $2, \ldots, N\}$. In (7), the prior distribution $p d f\left(\boldsymbol{\alpha}_{N E W}\right)$ is defined by (6). The conditional distribution $p d f\left(\mathbf{f}_{N E W} \mid \boldsymbol{\alpha}_{N E W}\right)$ is referred to as the likelihood function. It measures the probability of observing the new data $\left\{\left(\mathbf{x}^{(n)}, f_{N E W}^{(n)}\right) ; n=1,2, \ldots, N\right\}$.

To derive the likelihood function $p d f\left(\mathbf{f}_{N E W} \mid \boldsymbol{\alpha}_{N E W}\right)$, we assume that the modeling error $\varepsilon_{N E W}$ in (4) can be represented as a random variable with zero-mean Gaussian distribution:

$$
p d f\left(\varepsilon_{N E W}\right) \sim \operatorname{Gauss}\left(0, \sigma_{0}^{2}\right),
$$

where the standard deviation $\sigma_{0}$ indicates the magnitude of the modeling error. Similar to the parameter $\lambda$ in (5), the value of $\sigma_{0}$ can be determined by cross-validation [8]-[9]. Since the modeling error associated with the $n$-th data point $\left(\mathbf{x}^{(n)}, f_{N E W}{ }^{(n)}\right)$ is simply one sampling point of the random variable $\varepsilon_{N E W}$ that follows the Gaussian distribution in (8), the probability of observing the $n$-th data point is:

$$
\begin{aligned}
p d f\left(f_{N E W}^{(n)} \mid \boldsymbol{\alpha}_{N E W}\right) & =\frac{1}{\sqrt{2 \pi} \cdot \sigma_{0}} . \\
& \exp \left\{-\frac{1}{2 \cdot \sigma_{0}^{2}} \cdot\left[f_{N E W}^{(n)}-\sum_{k=1}^{K} \alpha_{N E W, k} \cdot b_{k}\left(\mathbf{x}^{(n)}\right)\right]^{2}\right\}
\end{aligned}
$$

Note that the likelihood function $p d f\left(f_{N E W}{ }^{(n)} \mid \boldsymbol{\alpha}_{N E W}\right)$ in (9) depends on the model coefficients $\left\{\alpha_{N E W, k} ; k=1,2, \ldots, K\right\}$. 
Assuming that all data points $\left\{\left(\mathbf{x}^{(n)}, f_{N E W}^{(n)}\right) ; n=1,2, \ldots, N\right\}$ are independently generated, we can write the likelihood function $p d f\left(\mathbf{f}_{N E W} \mid \boldsymbol{\alpha}_{N E W}\right)$ as:

$$
p d f\left(\mathbf{f}_{N E W} \mid \boldsymbol{\alpha}_{N E W}\right)=\prod_{n=1}^{N} p d f\left(f_{N E W}^{(n)} \mid \boldsymbol{\alpha}_{N E W}\right) .
$$

After the new data $\left\{\left(\mathbf{x}^{(n)}, f_{N E W}{ }^{(n)}\right) ; n=1,2, \ldots, N\right\}$ are available, the new coefficients $\left\{\alpha_{N E W, k} ; k=1,2, \ldots, K\right\}$ can be modeled by the probability density function $p d f\left(\boldsymbol{\alpha}_{N E W} \mid \mathbf{f}_{N E W}\right)$ (i.e., the posterior distribution) in (7). Depending on the shape of the posterior distribution $p d f\left(\boldsymbol{\alpha}_{N E W} \mid \mathbf{f}_{N E W}\right)$, the new coefficients $\left\{\alpha_{N E W, k} ; k=1,2, \ldots, K\right\}$ do not take all possible values with equal probability. If the posterior distribution $p d f\left(\boldsymbol{\alpha}_{N E W} \mid \mathbf{f}_{N E W}\right)$ reaches its maximum value at $\left\{\alpha^{*}{ }_{N E W, k} ; k=1\right.$, $2, \ldots, K\}$, these values $\left\{\alpha^{*}{ }_{N E W, k} ; k=1,2, \ldots, K\right\}$ are the optimal estimation of the new coefficients, since these coefficient values are most likely to occur. Such a method is referred to as the MAP estimation in the literature [8]-[9].

The aforementioned MAP estimation can be formulated as an optimization problem:

$$
\underset{\boldsymbol{a}_{N E W}}{\operatorname{maximize}} p d f\left(\boldsymbol{\alpha}_{N E W} \mid \mathbf{f}_{N E W}\right) \text {. }
$$

Substituting (5)-(7) and (9)-(10) into (11) and taking the logarithm for the merit function result in the following equivalent optimization formulation for MAP:

$$
\begin{aligned}
\underset{\boldsymbol{a}_{N E W}}{\operatorname{minimize}} \frac{\sigma_{0}^{2}}{\lambda^{2}} \sum_{k=1}^{K}\left(\frac{\alpha_{N E W, k}-\alpha_{O L D, k}}{\alpha_{O L D, k}}\right)^{2}+ \\
+\sum_{n=1}^{N}\left[f_{N E W}^{(n)}-\sum_{k=1}^{K} \alpha_{N E W, k} \cdot b_{k}\left(\mathbf{x}^{(n)}\right)\right]^{2}
\end{aligned}
$$

Studying (12) would reveal several important properties. First, the optimization in (12) is convex and, hence, can be solved both efficiently and robustly. Second, only the ratio $\sigma_{0} / \lambda$ is required to solve the new model coefficients $\left\{\alpha_{N E W, k} ; k=1,2\right.$, ..., $K\}$ from (12). Hence, we only need to determine the ratio $\sigma_{0} / \lambda$, instead of the individual parameters $\sigma_{0}$ and $\lambda$, by crossvalidation. Once the new model coefficients $\left\{\alpha_{N E W, k} ; k=1,2\right.$, ..., $K\}$ are found from (12), an updated indirect sensor model $f_{N E W}(\mathbf{x})$ in (4) is generated to match the new process condition after process shift occurs.

\section{Self-Healing Flow and Experimental Results}

In this section, we describe our proposed self-healing flow for a $25 \mathrm{GHz}$ differential Colpitts VCO example designed in a $32 \mathrm{~nm}$ CMOS SOI process. Fig. 1 shows the simplified schematic of the VCO. It consists of a cross-coupled differential pair connected to two common-gate Colpitts oscillators. The capacitor at the output is tunable so that the $\mathrm{VCO}$ frequency can be centered at different frequency bands. The bias voltage $V_{b}$ is controlled by a DAC for self-healing. More details about the VCO design can be found in [10].

For the VCO shown in Fig. 1, phase noise is an important performance of interest. Accurately measuring the phase noise at $25 \mathrm{GHz}$ is not trivial. Hence, indirect performance sensing is applied for on-chip phase noise measurement. Towards this goal, we identify four PoM metrics that are highly correlated with phase noise, as summarized in Table I. A quadratic model is fitted to predict the PoI (i.e., phase noise) from the PoM. In other words, the indirect sensor model $f(\mathbf{x})$ in (2) is a quadratic polynomial function. Once the model coefficients of $f(\mathbf{x})$ are determined, the quadratic model $f(\mathbf{x})$ is used for onchip phase noise estimation that is required for self-healing.

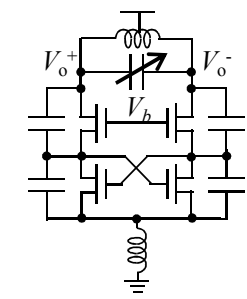

Fig. 1. Simplified circuit schematic is shown for a Colpitts VCO.

In this VCO example, the objective of self-healing is to find the optimal bias voltage to minimize phase noise. To this end, we implement a brute-force search algorithm where all possible bias voltages are set by the DAC and the phase noise values are estimated by the quadratic model $f(\mathbf{x})$ based on PoM. Next, the bias voltage corresponding to the minimum phase noise is chosen as the optimal solution.

Off-chip measurement data are collected from two silicon wafers and these data are used to perform an off-chip data analysis to validate the aforementioned self-healing flow. The first and second wafers contain 55 and 61 functional VCOs, respectively. Without self-healing, the parametric yield for these two wafers is $76.4 \%$ and $0 \%$, respectively. Note that there is a significant process shift between these two wafers, since the yield of the second wafer is substantially different from that of the first wafer.

In this example, since the parametric yield of the second wafer is extremely low, we want to apply self-healing to improve its yield. For testing and comparison purposes, four different self-healing methods are implemented:

- Ideal: The optimal bias voltage is determined by directly measuring the phase noise with an off-chip tester for all bias voltages. This approach is not considered as on-chip self-healing; however, it provides the upper bound of the yield improvement that can be achieved by self-healing.

- OLS-1: Traditional OLS is applied to fit the indirect sensor model based on the phase noise data measured by an off-chip tester for a few VCOs on the second wafer. In this case, no data from the first wafer is used. Next, the indirect sensor model is applied to the aforementioned selfhealing flow to heal all VCOs on the second wafer.

- OLS-2: Traditional OLS is applied to fit the indirect sensor model based on the phase noise data measured by an off-chip tester for all VCOs on the first wafer and a few VCOs on the second wafer. The indirect sensor model is then applied to self-heal all VCOs on the second wafer.

- BMF: An indirect sensor model is first fitted based on the measurement data from the first wafer. Next, the proposed BMF algorithm is applied to re-calibrate the old model and generate a new model based on the phase noise data 
measured by an off-chip tester for a few VCOs on the second wafer. The new model is applied to self-heal all VCOs on the second wafer.

Fig. 2 shows the parametric yield of the second wafer that is achieved by four different self-healing methods. Table II further summarizes the off-chip measurement cost for selfhealing. Studying Fig. 2 and Table II reveals several important observations. First, BMF requires substantially less VCOs measured by an off-chip tester to build the indirect phase noise sensor than the traditional OLS method. In this example, BMF needs to measure one VCO only, while both OLS-1 and OLS2 require to measure four VCOs $(4 \times)$ to achieve similar yield.

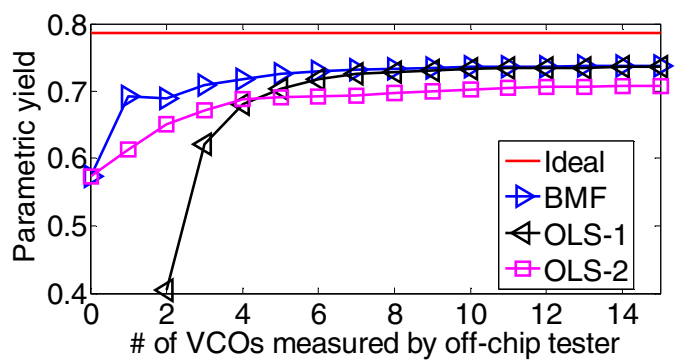

Fig. 2. Post-self-healing parametric yield of the second wafer is shown as a function of the number of VCOs from the second wafer measured by an offchip tester.

Table II

MEASUREMENT Cost AND PARAMETRIC YiELd FOR SELF-HEALING

\begin{tabular}{c|c|c}
\hline \hline Self-Healing Method & $\begin{array}{c}\text { \# of Measured VCOs } \\
\text { from Second Wafer }\end{array}$ & $\begin{array}{c}\text { Parametric Yield for } \\
\text { Second Wafer }\end{array}$ \\
\hline \hline Ideal & 61 & $78.69 \%$ \\
\hline OLS-1 & 4 & $68.06 \%$ \\
\hline OLS-2 & 4 & $68.78 \%$ \\
\hline BMF & 1 & $69.17 \%$ \\
\hline
\end{tabular}

Second, studying the BMF results in Fig. 2, we notice that if no measurement data is collected from the second wafer (i.e., the number of VCOs from the second wafer measured by an off-chip tester is zero) and the self-healing of the second wafer is performed with the indirect phase noise model fitted from the first wafer, the post-self-healing parametric yield of the second wafer is $59 \%$ only. Once a single VCO is measured for the second wafer, the indirect phase noise model is recalibrated by BMF and the post-self-healing yield is increased to $69.17 \%$. It, in turn, demonstrates that the aforementioned model re-calibration is a critical step for yield enhancement.

Third, the OLS-2 results in Fig. 2 demonstrate that if all measurement data from the first wafer are directly used by OLS, the fitted indirect sensor model is biased and does not accurately capture the shifted process condition associated with the second wafer. For this reason, the post-self-healing yield of OLS-2 remains lower than that of OLS-1, even if a large number of VCOs are measured for the second wafer. On the other hand, BMF does not suffer from such a bias issue, as it appropriately exploits the useful information from the first wafer through Bayesian inference.

Finally, the post-self-healing parametric yield of BMF is close to that of the "ideal" case. Compared to BMF, "ideal" self-healing increases the yield from $69.17 \%$ to $78.69 \%$. It implies that the modeling error of our proposed BMF method is reasonably small, even if a single VCO is measured from the second wafer. Fig. 3 further shows the scatter plot between the actual phase noise measured by an off-chip tester and the predicted phase noise estimated by BMF where the average modeling error is $0.56 \mathrm{dBc} / \mathrm{Hz}$.

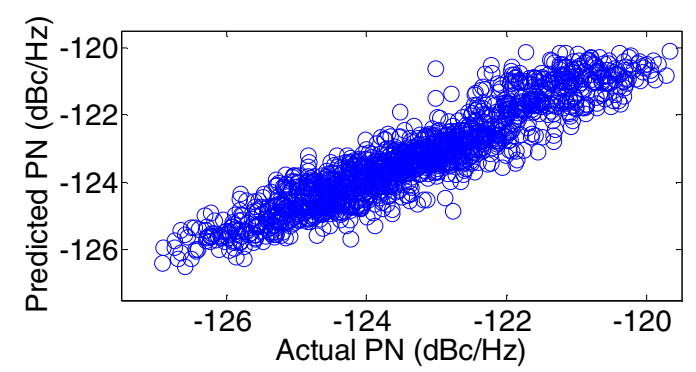

Fig. 3. Scatter plot is shown for the actual phase noise (measured by an offchip tester) and the predicted phase noise (estimated by the indirect phase noise sensor) for all VCOs on the second wafer.

\section{CONCLUSION}

In this paper, we propose a novel BMF technique to efficiently re-calibrate indirect performance sensors to accommodate the process shift associated with manufacturing lines. Towards this goal, a Bayesian inference is developed to accurately update the indirect sensor model by combining the old model coefficients with very few new measurement data. The proposed indirect performance sensing based on BMF is incorporated into an on-chip self-healing flow and is validated by off-chip data analysis for a $25 \mathrm{GHz} \mathrm{VCO}$ designed in a $32 \mathrm{~nm}$ CMOS SOI process.

\section{ACKNOWLEDGMENTS}

This work is sponsored by the DARPA HEALICS (Self-Healing MixedSignal Integrated Circuits) program under Air Force Research Laboratory (AFRL) contract FA8650-09-C-7924. The views expressed are those of the author and do not reflect the official policy or position of the Department of Defense or the U.S. Government.

\section{REFERENCES}

[1] S. Nassif, "Modeling and analysis of manufacturing variations," IEEE CICC, pp. 223-228, 2001.

[2] X. Li, et al., Statistical Performance Modeling and Optimization, Now Publishers, 2007.

[3] Semiconductor Industry Associate, International Technology Roadmap for Semiconductors, 2011.

[4] G. Keskin, et al., "Statistical modeling and post manufacturing configuration for scaled analog CMOS," IEEE CICC, 2010.

[5] C. Chien, et al., "Dual-control self-healing architecture for high performance radio SoC's," IEEE Design \& Test, 2013, to be published.

[6] S. Yaldiz, et al., "Indirect phase noise sensing for self-healing voltage controlled oscillators," IEEE CICC, 2011.

[7] B. Sadhu, et al., "A linearized, low-phase-noise VCO-based 25-GHz PLL with autonomic biasing," IEEE JSSC, vol. 48, no. 5, pp. 1138-1150, May 2013.

[8] C. Bishop, Pattern Recognition and Machine Learning, Prentice Hall, 2007.

[9] X. Li, et al., "Efficient parametric yield estimation of analog/mixedsignal circuits via Bayesian model fusion," IEEE ICCAD, pp. 627-634, 2012.

[10] J. Plouchart, et al., "A 23.5GHz PLL with an adaptively biased VCO in 32nm SOI-CMOS," IEEE TCAS-I, 2013, to be published. 